\title{
The challenge of mental health during Covid-19 outbreak: experience from metropolitan area of Milan
}

\author{
Federico Seghi $^{1}$ [ $\cdot$ Barbara Barbini $^{2} \cdot$ Linda Franchini $^{2} \cdot$ Cristina Colombo $^{1,2}$
}

Received: 1 June 2020 / Accepted: 16 June 2020 / Published online: 20 June 2020

c) Springer-Verlag GmbH Germany, part of Springer Nature 2020

After the outbreak of the novel coronavirus, recently named Sars-CoV-2, in Wuhan, China, Europe rapidly became the new epicenter for the virus spread. North of Italy, especially Lombardy, was the first European region where autochthonous cases were discovered, accounting for more than onethird of cases in Italy, with tremendous consequences on the common habits and lifestyle of the Italian population. In the absence of vaccines or specific therapeutics, the Government imposed national measures to reduce social interaction and ultimately limit the viral spread, including isolation, quarantine, and community containment [1]. The need to deal with a growing number of patients affected by severe respiratory symptoms, together with tight travel limitations, posed a great challenge to providing appropriate assistance to patients affected by other conditions, particularly mental disorders.

The effects of Covid-19 in the management of psychiatric conditions could be observed at different levels, both direct and indirect. In few weeks, the whole mental health system was severely remodeled and downsized [2]. With outpatient and day-care services limited to urgent visits that could not be deferred, most of the subjects with chronic disorders suddenly lost direct contact with their psychiatrist and related mental health-care professionals, causing profound limitations in daily routine, interpersonal contacts, and global functioning, possibly leading to distress and symptoms exacerbation. Experiences from previous epidemics and pandemics showed how social isolation itself could lead to anger, loneliness, and boredom, and might have a detrimental effect

Communicated by Andrea Schmitt.

Federico Seghi

seghi.federico@hsr.it

1 Università Vita-Salute San Raffaele, Via Olgettina 58, 20132 Milan, Italy

2 San Raffaele Turro Hospital, Mood Disorder Unit, Via Stamira D’Ancona 20, 20127 Milan, Italy on mental health, including acute stress disorder, mood and anxiety disorders, psychotic symptoms, and even suicidality [3], both in psychiatric patients and previously healthy subjects. Acutely ill psychiatric patients represent a further challenge to face, especially when in need of hospitalization. Disorganized behaviors, cognitive symptoms, and difficulties or complete lack of physical distancing due to crowded institutions increase the risk of viral transmission, also considering that the occurrence of psychopharmacological drug-related side effects may predispose to more severe Covid-19 manifestations [4].

As previously experienced in China [4], we had to adapt rapidly to these upcoming issues to preserve the general health of those patients referring to our psychiatric outpatient and inpatient services at San Raffaele Turro Hospital mood disorder Unit. Our services are integrated within the Milan metropolitan area, the one with the highest number of cases in Italy at the time of writing. We adopted multiple strategies, including telemedicine, restrictions to hospital access, basic preventive measures, and physical/laboratory monitoring of patients.

With the recent development of multiple tools in the field of telecommunication, telemedicine has already proved to be an effective strategy in the doctor-patient relationship, particularly in psychiatry [5]. The great majority of outpatients was promptly advised to remain home, unless in need of an urgent consultation. Our hospital provided a practical virtual platform to perform specialist assessments, with a generally positive response and thousands of patients who signed up in less than a month. We ensured that all the patients who had a scheduled appointment during the lockdown period were contacted by phone or other telematics platforms. The assessments were based on the evaluation of both mental health conditions and the impact of Covid-19 on everyday life. We used a structured interview, containing items that referred to understanding the lockdown measures, exacerbation of mood, anxiety and sleep symptoms, and economical repercussions of the emergency. We interviewed 179 
subjects in 2 months, $97.8 \%$ of which expressed satisfaction with the service offered.

In case of hospitalization, we remodeled the clinical routine and organization for inpatients to limit the spread of the virus within a high-risk environment, with the aim of zero infections among patients and health-care workers. Before the admission, every patient underwent a nasopharyngeal swab and a strict contact tracing, in cooperation with the Infectious Diseases staff; those who tested negative for SarsCoV-2 were admitted under a 3-day isolation regime. During the hospitalization, basic preventive measures were implemented, including frequent hand washing, physical distancing, and use of facemasks, with continuous training operated by the staff. Neither group activities nor visits from relatives were allowed, with the use of common rooms/spaces restricted to adequate distancing. Temperature, vitals, and respiratory symptoms were assessed daily on 39 consecutively admitted patients, which detected only 2 suspected cases. Those were quickly isolated and transferred to the Infectious Diseases ward; none of them were confirmed as Covid-19.

In line with general recommendations [6] and other reports from Lombardy [7, 8], we succeeded in consistently containing the viral transmission among our psychiatric population. Our experience appears crucial in determining measures that proved effective in the viral containment and allowed adequate care in a vulnerable population.

\section{Compliance with ethical standards}

Conflicts of interest Nothing to declare.

Ethics approval Not applicable.

\section{References}

1. Cetron M, Simone P (2004) Battling 21st-century scourges with a 14th-century toolbox. Emerg Infect Dis 10(11):2053-2054

2. de Girolamo G, Cerveri G, Clerici M, Monzani E, Spinogatti F, Starace F, Tura G, Vita A (2020) Mental health in the coronavirus disease 2019 emergency-the Italian response. JAMA Psychiatry. https://doi.org/10.1001/jamapsychiatry.2020.1276

3. Xiang YT, Yang Y, Li W et al (2020) Timely mental health care for the 2019 novel coronavirus outbreak is urgently needed. Lancet Psychiatry 7(3):228-229

4. Xiang YT, Zhao YJ, Liu ZH et al (2020) The COVID-19 outbreak and psychiatric hospitals in China: managing challenges through mental health service reform. Int J Biol Sci 15 16(10):1741-1744

5. Norman S (2006) The use of telemedicine in psychiatry. J Psychiatr Ment Health Nurs 13(6):771-777

6. Starace F, Ferrara M (2020) COVID-19 disease emergency operational instructions for mental health departments issued by the Italian society of epidemiological psychiatry. Epidemiol Psychiatr Sci 31(29):e116

7. D'Agostino A, Demartini B, Cavallotti S, Gambini O (2020) Mental health services in Italy during the COVID-19 outbreak. Lancet Psychiatry 7(5):385-387

8. Percudani M, Corradin M, Moreno M, Indelicato A, Vita A (2020) Mental health services in lombardy during COVID-19 outbreak. Psychiatry Res 288:112980

Funding Not applicable.

Data availability Not applicable. 